\title{
An admission control model considering handover cost for LTE networks
}

\author{
Bin Zeng and Lu Yao \\ Department of Management, Naval University of Engineering, Wuhan, Hubei, 430033, \\ China \\ E-mail: zbtrueice@163.com \\ www.hjgcdx.cn
}

\begin{abstract}
An approach is presented to manage and support a LTE wireless communication networks. The achievements are expressed in terms of formulation and performance. In terms of formulation, a mathematical expression is modeled to describe the Admission Control and handover in LTE networks problem. The outcome of the work will be helpful for system subscribers to make integrated decision and maintain the system. Owing to the experience, Lagrangean relaxation and subgradient method are adopted as the main solution algorithm. In terms of performance, our Lagrangean relaxation based solution has more significant improvement than other intentional algorithm.
\end{abstract}

Keywords: Handover; Long Term Evolution; Mobile communication; Quality of service; Admission Control.

\section{Introduction}

Emerging requirements for higher rate data services and better spectrum efficiency are the main drivers identified for the third generation mobile radio systems. Fourth Generation (4G) LTE networks will allow subscribers to experience new and unique offerings that provide them with enhanced multimedia [1], customization, roaming, voice, location-enabled, and other capabilities to meet the ever increasing expectations of wireless communications. Key objectives of the development and deployment of 4G LTE include: (1) high-speed transmission; (2) high-quality services; (3) unique and enhanced service capabilities [2]; (4) ease of operation and maintenance; (5) flexibility to add services.

In order to maintain the quality of the radio connections, an admission control is needed to prevent the system from getting overloaded [3]. In this paper the uplink part of the Admission Control is considered. The uplink admission control strategy can be based on the received wide band interference levels. If the interference level gets too high, no new users are admitted. The 
Admission Control allows a new user into the radio access network if the admission does not cause an excessive interference in the system [4]. The target of the uplink Admission Control is to prevent the overload of the LTE system and to guarantee the quality of the existing connections and to guarantee the planned coverage area of the system. Before a new user is admitted to the system, the Admission Control algorithm must calculate the increase in the total interference level due to a new user.

\section{Admission Control Model}

We consider not only the new mobile terminals in this problem but also the existing mobile terminals in admission control model. The new mobile terminal in this model can be handed to one of the base station that could provide service to it or be blocked. The existing mobile terminals in this model could be handed over to the base stations that can serve it. To ensure the QoS of the existing mobile terminals, they could be blocked $[5,6]$. We might admit more new calls while handover the existing mobile terminals to another service base station. However, we should take both the handover cost and admitted revenue into account. The ratio of the handoff cost of existing mobile terminals from currently assigned base station to another base station and the total revenue of admittance of new mobile terminals into the system not exceed a given bound $U$.

We also do not concern the mobility of the existing mobile terminals in our formulation, but our model could be extended for considering mobility of mobile terminals. If we take mobility into account, the new terminals could be divided into handoff calls and real new calls. Since the model is non-preemptive, continuous-time and call arrival is poisson distribution, we can assign the handoff calls higher priority than the real new calls when the new calls arrive at the same time. After handling the handoff calls, the real new calls will be processed. The problem description is shown as follow: Given the candidate base station (BS) location, radius of each base station (BS), the distribution of users, the users' homing status. And the ratio upper bound of handoff cost and revenue. The objective is to maximize the total revenue of admittance of new mobile terminals into the system.

\subsection{Notation of Parameters and Decision Variable}

The notations of the model parameters are given as below. $B$ denotes the set of candidate locations for base stations. $S$ denotes the power that a base station received from a mobile terminal that is homed to the base station with perfect power control. $T$ denotes the set of mobile terminals. $W$ denotes the set of total 
bandwidth. $E_{b}$ denotes the energy that BS received. $N_{\text {Total }}$ denotes Total noise. $\alpha$ denotes the voice activity. $M_{j}$ denotes the upper bound on the number of users that can active at the same time at base station $\mathrm{j} . C_{j}$ denotes the number of users who can be active at the same time in the base station $j . \tau$ denotes attenuation actor. $D_{j t}$ denotes the distance between base station $j$ and mobile terminal $t . D_{j j}$ ' denotes Distance between base station $j$ and $j^{\prime}$. $N_{0}$ denotes the background noise. $K_{R}$ denotes the data bit rate. $I_{j}$ denotes the intracell interference. $I_{j j}$, denotes the interference from base station $j$ ' to $j$. $\mu_{j t}$ denotes the indicator function which is 1 if mobile terminal $\mathrm{t}$ can be served by base station $j$ and 0 otherwise. $R_{j}$ denotes the upper bound of radius of base station $j$. $G$ denotes the processing gain. $\alpha_{t}$ denotes the revenue from admitting mobile terminal $t \in T$ into to the system. $T^{\prime}$ denotes the set of existing mobile terminals. $T$ ' ' denotes the set of new mobile terminals whose admittance into the cell is to be determined. $b$ ' denotes The artificial base station to carry the rejected call when Admission Control function decides to reject the call. $B^{\prime}$ denotes the set of $B \cup\left\{b^{\prime}\right\} . Y_{j}$ denotes the set of transmission radius of base station $j . f_{t}$ denotes the cost of handoff from currently assigned base station to base station $t . b_{t}$ denotes the base station which $t \in T$, was originally assigned. $r_{j}$ denotes the transmission radius of base station $j . U$ denotes The prespecified threshold of the ratio of the handoff cost of existing mobile terminals from currently assigned base station to another base station and the total revenue of admittance of new mobile terminals. $z_{j t}$ denotes the decision variable which is 1 if mobile terminal $t$ is serviced by base station $j$ and 0 otherwise.

\subsection{Problem Formulation}

Objective function:

$$
\begin{aligned}
& Z_{I P}=\max \left(\sum_{t \in T^{\prime \prime}} a_{t} \sum_{j \in B} z_{j t}-\sum_{t \in T^{\prime}} f_{t} \sum_{j^{\prime} \in B-\left\{b_{t}\right\}} z_{j^{\prime} t}\right) \\
& =\min \left(-\left(\sum_{t \in T^{\prime \prime}} a_{t} \sum_{j \in B} z_{j t}-\sum_{t \in T^{\prime}} f_{t} \sum_{j^{\prime} \in B-\left\{b_{t}\right\}} z_{j^{\prime} t}\right)\right)
\end{aligned}
$$

subject to:

$$
\begin{gathered}
\left(\frac{E_{b}}{N_{\text {total }}}\right)_{\text {req }} \leq \frac{\frac{S}{N_{0}}}{1+\frac{1}{G} \alpha \frac{S}{N_{0}}\left(\sum_{t \in T} z_{j t}-1\right)+\frac{1}{G} \alpha \frac{S}{N_{0}} \sum_{\substack{j^{\prime} \in B \\
j^{\prime} \neq j}} \sum_{t \in T}\left(\frac{D_{j^{\prime} t}}{D_{j t}}\right)^{\tau} z_{j^{\prime} t}} \forall j \in B \\
\sum_{t \in T} z_{j t} \leq M_{j}
\end{gathered}
$$




$$
\begin{array}{cr}
D_{j t} z_{j t} \leq R_{j} \mu_{j t} & \forall j \in B, t \in T \text { (3) } \\
z_{j t} \leq \mu_{j t} & \forall j \in B, t \in T \text { (4) } \\
\sum_{j \in B^{\prime}} z_{j t}=1 & \forall t \in T^{\prime \prime} \text { (5) } \\
\sum_{j \in B} z_{j t}=1 & \forall t \in T^{\prime} \text { (6) } \\
\sum_{t \in T^{\prime}} f_{t} \sum_{j^{\prime} \in B-\left\{b_{t}\right\}} z_{j^{\prime} t} \\
\sum_{t \in T^{\prime \prime}} a_{t} \sum_{j \in B} z_{j t} & \\
z_{j t}=0 \text { or 1 } &
\end{array}
$$

The objective function is to maximize the total revenue of admittance of new mobile terminals into the system. $a_{t}$ is the average revenue of each new mobile terminal and $a_{t}$ is the average handing over cost of each existing mobile terminal. In terms of convenience, we can translate the problem into an equivalent description that is to minimize the negative total revenue. Constraint (1) is to ensure that each mobile terminal is served with its base station with required QoS. The left hand side of this inequality is the minimal SIR (Signalto-Interference Ratio) which each connection should be hold. The right hand side means the real SIR. The denominator of the right hand side is the total interference value, including white noise, the intra-cell interference, and intercell interference. Constraint (2) is to ensure that the number of users who can be active at the same time in a base station would not exceed the base station's upper bound. Constraint (3) is to ensure that a base station can only serve the mobile terminals inside its coverage area of effective radius. Constraint (4) is to ensure that if a base station does not provide service to a mobile terminal, then the decision variable $z_{j t}$ must equal to 0 . Constraint (5) is to ensure that each new mobile terminal who wants to be active can be homed to only one physical base station or be rejected. Constraint (6) is to ensure that each existing mobile terminal should be homed to only one physical base station. Constraint (7) is to ensure that the ratio of the handoff cost of existing mobile terminals from currently assigned base station to another base station and the total revenue of admittance of new mobile terminals into the system do not exceed a given bound $U$. Constraint (8) is to enforce the integer property of the decision variables. 


\section{Lagrangean Relaxation Based Algorithm}

The Lagrangean relaxation based algorithm is used to deal with the "Admission Control Model" problem. We call it LR. Shown as follow:

- Step1. Read configuration file to construct BSs and MTs.

- Step2. Calculate constant parameters, such as $\delta_{j t}, D_{j t}$ and assign Lagrangean relaxation improve counter to equal 20.

- Step3. Initialize multipliers.

- Step4. According to given multipliers, optimally solve these subproblem to get the value of Lagrangean dual problems.

- Step5. Get the total revenue, the value of $Z_{I P}$.

-Step6. If $Z_{I P}$ is smaller than $Z_{I P^{*}}$, we assign $Z_{I P^{*}}$ to equal $Z_{I P}$. Otherwise, we minus 1 from the improve counter.

-Step7. Calculate step size and adjust Lagrangean relaxation multipliers.

-Step8. Iteration counter increases 1. If interaction counter is over threshold of system, stop this program. And, $Z_{I P *}$ is our best solution. Otherwise, repeat Step4.

In order to review our results, we develop a simple algorithm (Simp) to test our work. In simple algorithm, we just assign each mobile terminal to be serviced by the base station that has the shortest distance between them. We refer it as Simp to compare the experimental results of LR.

\section{Computational Experiment}

The parameters are as follows: $S / N_{0}=7 \mathrm{db} . E_{b} / N_{\text {total }}=6.58 \mathrm{db}$. Voice communication bit rate $=9.6 \mathrm{Kbps}$. Voice activity $=0.5$. Signal attenuation $=4$. Maximum number of users who can be active at the same time to a base station $=120$. Average revenue cost of a new mobile terminal user $=10$ units. Fixed cost of handing over an existing mobile terminal user $=5$ units. In this case, we have 8 BSs, 50 existing mobile terminals and 200 new mobile terminals.

Tab. 1 The Result of Case for our model. Unit: Total Revenue (NTD.)

\begin{tabular}{|l|l|l|l|l|l|l|l|l|l|l|}
\hline & 1 & 2 & 3 & 4 & 5 & 6 & 7 & 8 & 9 & 10 \\
\hline Simp & 1640 & 1730 & 1660 & 1640 & 1660 & 1660 & 1450 & 1680 & 1590 & 1570 \\
\hline LR & 1665 & 1765 & 1700 & 1665 & 1700 & 1765 & 1600 & 1715 & 1630 & 1630 \\
\hline Improvement to Simp & 1.50 & 1.98 & 2.35 & 1.50 & 2.35 & 5.95 & 9.38 & 2.04 & 2.45 & 3.68 \\
\hline
\end{tabular}

\section{Conclusion}

The objective of our work is to regulate the operation of a network in such a way that ensures the uninterrupted service provision to the existing connections and 
at the same time accommodates in an optimum way the new connection requests. This is done by managing the available network resources and allocating them in an optimum way among the system users.

\section{References}

1. X. Xiang, C. Lin, X. Chen, X. Shen, Toward Optimal Admission Control and Resource Allocation for LTE-A Femtocell Uplink, IEEE Transactions on Vehicular Technology 64, 3247 (2015).

2. F Zarai, KB Ali, MS Obaidat, Adaptive call admission control in 3GPP LTE networks, Int J. Comm Sys 27, 1522 (2014).

3. SP Chung, YW Chen, Performance analysis of call admission control in SFR-based LTE systems, IEEE Communications Letters 16, 1014(2012).

4. TFZ. Badri, R. Saadane, S. Mbarki, Call Admission Control Scheme and Handover Management in LTE Femtocell-Macrocell Integrated Networks, Computer and Information Science 8, 135(2015).

5. N Sinclair, D Harle, IA Glover, J Irvine, An Advanced SOM Algorithm Applied to Handover Management Within LTE, IEEE Transactions on Vehicular Technology 62, 1883(2013).

6. $\mathrm{P} \mathrm{Mu}, \mathrm{R}$ Barco, S Fortes, Conflict resolution between load balancing and handover optimization in LTE networks, IEEE Communications Letters 18, 1795(2014). 